\title{
Basu's Theorem
}

\author{
Malay Ghosh
}

Professor Basu, in his long illustrious career, has made many fundamental contributions to the foundations of statistical inference. Among others, I point out his work on ancillarity, likelihood principle, partial and marginal sufficiency, randomization and foundations of survey sampling.

In spite of all the above contributions, Basu is possibly the most well-known to a vast majority of statisticians for a theorem which bears his name. Basu's Theorem, published in Sankhya, 1955, has served several generations of statisticians as a fundamental tool for proving independence of statistics. The theorem itself is beautiful because of its elegance and simplicity, and yet one must acknowledge its underlying depth, as it is built on several fundamental concepts of statistics, such as sufficiency, completeness and ancillarity.

The theorem simply states that if a sufficient statistic $T$ is boundedly complete and a statistic $U$ is ancillary, then $T$ and $U$ are independently distributed. But the theorem is not just useful for what it says. It can be used in a wide range of applications such as in distribution theory, hypothesis testing, theory of estimation, calculation of moments of many complicated statistics, calculation of mean squared errors of empirical Bayes estimators, and even surprisingly, establishing infinite divisibility of certain distributions. The application possibly extends to many other areas of statistics which I have not come across. I strongly believe that even probabilists can benefit by knowing this theorem, since it may provide a handy tool for finding distributions of many complex statistics.

A detailed set of examples showing applications of Basu's Theorem in various branches of statistics is available in Ghosh (2002). I will present only a few of them here. But first I will discuss a few conceptual issues as pointed out in Lehmann (1981) and DasGupta (2006).

Lehmann (1981) pointed out that the properties of minimality and completeness of a sufficient statistic are of a rather different nature. A complete sufficient statistic is minimal sufficient, but the converse is not necessarily true. The existence of a minimal sufficient statistic $T$, by itself, does not guarantee that there does not exist any function of $T$ which is ancillary. Basu's Theorem tells us that if $T$ is complete in addition to being sufficient, then no ancillary statistic other than the constants can be computed from $T$. Thus, by Basu's Theorem, completeness of a sufficient statistic $T$ characterizes the success of $T$ in separating the informative part of the data from that part, which by itself, carries no information. The following example illustrates this.

Example 1 Let $X_{1}, \cdots, X_{n}$ be independent and identically distributed (iid) with joint probability density function (pdf) belonging to the double exponential family

M. Ghosh $(\bowtie)$

Distinguished Professor of Statistics, University of Florida, Gainesville, FL 32611

e-mail: ghoshm@stat.ufl.edu 


$$
\mathcal{P}=\left\{\prod_{i=1}^{n} f_{\theta}\left(x_{i}\right): f_{\theta}(x)=f(x-\theta), x \in \mathcal{R}^{1}, \theta \in \mathcal{R}^{1}\right\}
$$

where $f(x)=(1 / 2) \exp (-|x|)$. Let $X_{(1)} \leq X_{(2)} \leq \cdots \leq X_{(n)}$ denote the ordered $X_{i}$ 's. Then $T=\left(X_{(1)}, \cdots, X_{(n)}\right)$ is minimal sufficient for $\mathcal{P}$. But clearly $T$ is not boundedly complete as can be verified directly by showing that the expectation of any bounded function of $X_{(n)}-X_{(1)}$ (with the exception of constants) is a nonzero constant not depending on $\theta$, but that the probability that the function is equal to that constant is not 1 (in fact equal to zero). In this example, $X_{(n)}-X_{(1)}$, while ancillary, is not independent of $T$. On the other hand, if one considers instead the augmented class of all continuous pdf's $\mathcal{P}=\left\{\prod_{i=1}^{n} f\left(x_{i}\right): f\right.$ continuous $\}$, then $T$ is indeed complete, and Basu's Theorem asserts that there does not exist any non-constant function of $T$ which is ancillary.

Thus, for the double exponential family, sufficiency has not been successful in "squeezing out" all the ancillary material, while for the augmented family, success takes place by virtue of Basu's Theorem.

There are several ways to think of possible converses to Basu's Theorem. One natural question is that if $T$ is boundedly complete and sufficient, and $U$ is distributed independently of $T$ for every $\theta \in \Theta$, then is $U$ ancillary? The answer is no as pointed out by in Koehn and Thomas (1975) in the following example.

Example 2 Let $X \sim$ uniform $[\theta, \theta+1)$, where $\theta \in \Theta=\{0, \pm 1, \pm 2, \cdots$,$\} . Then X$ has pdf $f_{\theta}(x)=$ $I_{[[x]=\theta]}$, where $[x]$ denotes the integer part of $x$. It is easy to check that $[X]$ is complete sufficient for $\theta \in \Theta$, and is also distributed independently of $X$, but clearly $X$ is not ancillary!

The above apparently trivial example brings out several interesting issues. First, since $P_{\theta}([X]=$ $\theta)=1$ for all $\theta \in \Theta$, so that $[X]$ is degenerate with probability 1 . Indeed, in general, a nontrivial statistic cannot be independent of $X$, because if this were the case, it would be independent of every function of $X$, and thus independent of itself! However, this example shows also that if there exists a nonempty proper subset $\mathcal{X}_{0}$ of $\mathcal{X}$, and a nonempty proper subset $\Theta_{0}$ of $\Theta$ such that

$$
\begin{aligned}
P_{\theta}\left(\mathcal{X}_{0}\right) & =1 \text { for } \theta \in \Theta_{0} ; \\
& =0 \text { for } \theta \in \Theta-\Theta_{0},
\end{aligned}
$$

then the converse to Basu's Theorem may fail to hold. In Example 2, $\mathcal{X}=\mathcal{R}^{1}$, and $\Theta$ is the set of all integers. Taking $\Theta_{0}=\left\{\theta_{0}\right\}$ and $\mathcal{X}_{0}=\left[\theta_{0}, \theta_{0}+1\right)$, one produces a counterexample to a possible converse to Basu's Theorem.

Basu (1958) gave a sufficient condition for the converse to his theorem. First he defined two probability measures $P_{\theta}$ and $P_{\theta^{\prime}}$ to be overlapping if they do not have disjoint supports. In Example 2, all probability measures $P_{\theta}, \theta \in \Theta$ are non-overlapping. The family $\mathcal{P}$ is said to be connected if for every pair $\left\{\theta, \theta^{\prime}\right\}, \theta \in \Theta, \theta^{\prime} \in \Theta$, there exist $\theta_{1}, \cdots, \theta_{k}$, each belonging to $\Theta$ such that any two members of the sequence overlap. The following theorem is given in Basu (1958).

Theorem 1 Let $\mathcal{P}=\left\{P_{\theta}, \theta \in \Theta\right\}$ be connected, and $T$ be sufficient for $\mathcal{P}$. Then $U$ is ancillary if $T$ and $U$ are independent for every $\theta \in \Theta$.

It is only the sufficiency and not the completeness of $T$ which plays a role in Theorem 1 . An alternative way to think about a possible converse to Basu's Theorem is whether the independence of all ancillary statistics with a sufficient statistic $T$ implies that $T$ is boundedly complete. The answer is again NO as Lehmann (1981) produces the following counterexample. 
Example 3 Let $X$ be a discrete random variable assuming values $x$ with probabilities $p(x)$ as given below:

\begin{tabular}{lcccccccccc}
\hline$x$ & -5 & -4 & -3 & -2 & -1 & 1 & 2 & 3 & 4 & 5 \\
$p(x)$ & $\alpha^{\prime} p^{2} q$ & $\alpha^{\prime} p q^{2}$ & $\frac{1}{2} p^{3}$ & $\frac{1}{2} q^{3}$ & $\gamma^{\prime} p q$ & $\gamma p q$ & $\frac{1}{2} q^{3}$ & $\frac{1}{2} p^{3}$ & $\alpha p q^{2}$ & $\alpha p^{2} q$ \\
\hline
\end{tabular}

Here $0<p=1-q<1$, is the unknown parameter, and $\alpha, \alpha^{\prime}, \gamma, \gamma^{\prime}$ are known positive constants satisfying $\alpha+\gamma=\alpha^{\prime}+\gamma^{\prime}=3 / 2$. In this example, $|X|$ is minimal sufficient, $P(X>0)=1 / 2$ so that $U=I_{[X>0]}$ is ancillary. However, if $\alpha \neq \alpha^{\prime}$, then $U$ is not distributed independently of $T$.

Lehmann (1981) pointed out that this converse to Basu's Theorem fails to hold because ancillarity is a property of the whole distribution of a statistic, while completeness is a property dealing only with expectations. He showed also that correct versions of the converse could be obtained either by replacing ancillarity with the corresponding first order property or completeness with a condition reflecting the whole distribution.

To this end, define a statistic $V \equiv V(X)$ to be first order ancillary if $E_{\theta}(V)$ does not depend on $\theta \in \Theta$. Then one has a necessary and sufficient condition for Basu's Theorem.

Theorem 2 A necessary and sufficient condition for a sufficient statistic $T$ to be boundedly complete is that every bounded first order ancillary $V$ is uncorrelated with every bounded real-valued function of $T$ for every $\theta \in \Theta$.

An alternative approach to obtain a converse is to modify instead the definition of completeness. Quite generally, a sufficient statistic $T$ is said to be $\mathcal{G}$-complete ( $\mathcal{G}$ is a class of functions) if for every $g \in \mathcal{G}, E_{\theta}[g(T)]=0$ for all $\theta \in \Theta$ implies that $P_{\theta}[g(T)=0]=1$ for all $\theta \in \Theta$. Suppose, in particular, $\mathcal{G}=\mathcal{G}_{0}$, where $\mathcal{G}_{0}$ is the class of all two-valued functions. Then Lehmann (1981) proved the following theorem.

Theorem 3 Suppose $T$ is sufficient and every ancillary statistic $U$ is distributed independently of $T$. Then $T$ is $\mathcal{G}_{0}$-complete.

Basu's Theorem implies the independence of $T$ and $U$ when $T$ is boundedly complete and sufficient, while $U$ is ancillary. This, in turn, implies the $\mathcal{G}_{0}$-completeness of $T$. However, the same Example 3 shows that neither of the reverse implications is true. On the other hand, if instead of $\mathcal{G}_{0}$, one considers $\mathcal{G}_{1}$ which are conditional expectations of all two-valued functions with respect to a sufficient statistic $T$, then Lehmann proved the following theorem.

Theorem 4 A necessary and sufficient condition for a sufficient statistic $T$ to be $\mathcal{G}_{1}$-complete is that every ancillary statistic $U$ is independent of $T$ (conditionally) for every $\theta \in \Theta$.

Theorems 2-4, provide conditions under which a sufficient statistic $T$ has some form of completeness (not necessarily bounded completeness) if it is independent of every ancillary $U$. However, Theorem 1 says that ancillarity of $U$ does not follow even if it is independent of a complete suffcient statistic. As shown in Example 2, $[X]$ is complete sufficient, and hence, by Basu's Theorem, is independent of every ancillary $U$, but $[X]$ independent of $X$, but $X$ not ancillary.

Next we provide a few selected examples which show multifaceted applications of Basu's Theorem.

Example 4 (A Distribution Theory Result) Let $\boldsymbol{X}_{i}=\left(X_{1 i}, X_{2 i}\right)^{T}$ be $n$ iid random variables, each having a bivariate normal distribution with means $\mu_{1}\left(\in \mathcal{R}^{1}\right)$ and $\mu_{2}\left(\in \mathcal{R}^{1}\right)$, variances $\sigma_{1}^{2}(>0)$ and $\sigma_{2}^{2}(>0)$, and correlation $\rho \in(-1,1)$. Let $\bar{X}_{j}=n^{-1} \sum_{i=1}^{n} X_{j i}, S_{j}^{2}=\sum_{i=1}^{n}\left(X_{j i}-\bar{X}_{j}\right)^{2}(j=1,2)$ and $R=\sum_{i=1}^{n}\left(X_{1 i}-\bar{X}_{1}\right)\left(X_{2 i}-\bar{X}_{2}\right) /\left(S_{1} S_{2}\right)$. Under the null hypothesis $H_{0}: \rho=0,\left(\bar{X}_{1}, \bar{X}_{2}, S_{1}^{2}, S_{2}^{2}\right)$ is complete sufficient for $\left(\mu_{1}, \mu_{2}, \sigma_{1}^{2}, \sigma_{2}^{2}\right)$, while $R$ is ancillary. Thus $\left(\bar{X}_{1}, \bar{X}_{2}, S_{1}^{2}, S_{2}^{2}\right)$ is distributed independently of $R$ when $\rho=0$. Due to the mutual independence of $\bar{X}_{1}, \bar{X}_{2}, S_{1}^{2}$ and $S_{2}^{2}$ when 
$\rho=0$, one gets now the mutual independence of $\bar{X}_{1}, \bar{X}_{2}, S_{1}^{2}, S_{2}^{2}$ and $R$ when $\rho=0$, and the joint pdf of these five statistics is now the product of the marginals. Now to derive the joint pdf $q_{\mu_{1}, \mu_{2}, \sigma_{1}^{2}, \sigma_{2}^{2}, \rho}\left(\bar{x}_{1}, \bar{x}_{2}, s_{1}^{2}, s_{2}^{2}, r\right)$ of these five statistics for an arbitrary $\rho \in(-1,1)$, by the Factorization Theorem of sufficiency, one gets

$$
q_{\mu_{1}, \mu_{2}, \sigma_{1}^{2}, \sigma_{2}^{2}, \rho}\left(\bar{x}_{1}, \bar{x}_{2}, s_{1}^{2}, s_{2}^{2}, r\right)=q_{0,0,1,1,0}\left(\bar{x}_{1}, \bar{x}_{2}, s_{1}^{2}, s_{2}^{2}, r\right) \frac{L\left(\mu_{1}, \mu_{2}, \sigma_{1}^{2}, \sigma_{2}^{2}, \rho\right)}{L(0,0,1,1,0)},
$$

where $L(\cdot)$ denotes the likelihood function under the specified values of the parameters.

Example 5 This example, taken from Boos and Hughes-Oliver (BH) (1998), is referred to as the Monte Carlo Swindle. The latter refers to a simulation technique that ensures statistical accuracy with a smaller number of replications at a level which one would normally expect from a much larger number of replications. Johnstone and Velleman (1985) provide many such examples. One of their examples taken by $\mathrm{BH}$ shows that if $M$ denotes a sample median in a random sample of size $n$ from a $N\left(\mu, \sigma^{2}\right)$ distribution, then the Monte Carlo estimate of $V(M)$ requires a much smaller sample size to attain a prescribed accuracy, if instead one finds the Monte Carlo estimate of $V(M-\bar{X})$ and adds the usual estimate of $\sigma^{2} / n$ to the same.

We do not provide the detailed arguments of BH to demonstrate this. We point out only the basic identity $V(M)=V(M-\bar{X})+V(\bar{X})$ as used by these authors. As noticed by $\mathrm{BH}$, this is a simple consequence of Basu's Theorem. As mentioned in Example 2, for fixed $\sigma^{2}, \bar{X}$ is complete sufficient for $\mu$, while $M-\bar{X}=\operatorname{med}\left(X_{1}-\mu, \cdots, X_{n}-\mu\right)-(\bar{X}-\mu)$ is ancillary. Hence, by Basu's Theorem,

$$
V(M)=V(M-\bar{X}+\bar{X})=V(M-\bar{X})+V(\bar{X})=V(M-\bar{X})+\sigma^{2} / n .
$$

Hogg and Craig (1956) have provided several interesting applications of Basu's Theorem. Among these, there are some hypothesis testing examples where Basu's Theorem aids in the derivation of the exact distribution of $-2 \log _{e} \lambda$ under the null hypothesis $H_{0}, \lambda$ being the generalized likelihood ratio test (GLRT) statistic. One common feature in all these problems is that the supports of all the distributions depend on parameters. We discuss one of these examples in its full generality.

Example 6 Let $X_{i j}\left(j=1, \cdots, n_{i} ; i=1, \cdots, k\right)(k \geq 2)$ be mutually independent, $X_{i j}(j=$ $\left.1 . \cdots, n_{i}\right)$ being iid with common pdf

$$
f_{\theta_{i}}\left(x_{i}\right)=\left[h\left(x_{i}\right) / H\left(\theta_{i}\right)\right] I_{\left[0 \leq x_{i} \leq \theta_{i}\right]}, \quad i=1, \cdots, k,
$$

where $H(u)=\int_{0}^{u} h(x) d x$, and $h(x)>0$ for all $x>0$. We want to test $H_{0}: \theta_{1}=\cdots=\theta_{k}$ against the alternative $H_{1}$ : not all $\theta_{i}$ are equal. We write $\boldsymbol{X}_{i}=\left(X_{i 1}, \cdots, X_{i n_{i}}\right)^{T}, i=1, \cdots, k$ and $\boldsymbol{X}=\left(\boldsymbol{X}_{1}^{T}, \cdots, \boldsymbol{X}_{k}^{T}\right)^{T}$. Also, let $T_{i} \equiv T_{i}\left(\boldsymbol{X}_{i}\right)=\max \left(X_{i 1}, \cdots, X_{i n_{i}}\right), i=1, \cdots, k$, and $T=$ $\max \left(T_{1}, \cdots, T_{k}\right)$. The unrestricted MLE's of $\theta_{1}, \cdots, \theta_{k}$ are $T_{1}, \cdots, T_{k}$. Also, under $H_{0}$, the MLE of the common $\theta_{i}$ is $T$. Then the GLRT statistic for testing $H_{0}$ against $H_{1}$ simplifies to $\lambda(\boldsymbol{X})=$ $\prod_{i=1}^{k} H^{n_{i}}\left(T_{i}\right) / H^{n}(T)$, where $n=\sum_{i=1}^{k} n_{i}$. Hence,

$$
\begin{aligned}
-2 \log _{e} \lambda(\boldsymbol{X}) & =\sum_{i=1}^{k}\left[-2 \log _{e}\left\{H^{n_{i}}\left(T_{i}\right) / H^{n_{i}}(\theta)\right\}\right] \\
& -\left[-2 \log _{e}\left\{H^{n}(T) / H^{n}(\theta)\right\}\right],
\end{aligned}
$$

where $\theta$ denotes the common unknown value of the $\theta_{i}$ 's under $H_{0}$. It follows from (4) that $T_{1}, \cdots, T_{k}$ are independent with distribution functions $H^{n_{i}}\left(t_{i}\right) / H^{n_{i}}\left(\theta_{i}\right),(i=1, \cdots, k)$. Hence, under $H_{0}$, $H^{n_{i}}\left(T_{i}\right) / H^{n_{i}}(\theta)$ are iid uniform $(0,1)$. Accordingly, under $H_{0}$, 


$$
\sum_{i=1}^{k}\left[-2 \log _{e}\left\{H^{n_{i}}\left(T_{i}\right) / H^{n_{i}}(\theta)\right\}\right] \sim \chi_{2 k}^{2}
$$

Also, under $H_{0}$, the distribution function of $T$ is $H^{n}(t) / H^{n}(\theta)$, and hence, $H^{n}(T) / H^{n}(\theta)$ is uniform $(0,1)$ under $H_{0}$. Thus, under $H_{0}$,

$$
-2 \log _{e}\left[H^{n}(T) / H^{n}(\theta)\right] \sim \chi_{2}^{2} .
$$

So far, we have not used Basu's Theorem. In order to use it, first we observe that under $H_{0}, T$ is complete sufficient for $\theta$, while $\lambda$ is ancillary. Hence, under $H_{0}, T$ is distributed independently of $-2 \log _{e} \lambda$. Also, from (5),

$$
\sum_{i=1}^{k}\left[-2 \log _{e}\left\{H^{n_{i}}\left(T_{i}\right) / H^{n_{i}}(\theta)\right\}\right]=\left[-2 \log _{e} \lambda\right]+\left[-2 \log _{e}\left\{H^{n}(T) / H^{n}(\theta)\right\}\right] .
$$

The two components in the right hand side of (8) are independent. Now by (6), (7) and the result that if $W_{1}$ and $W_{2}$ are independent with $W_{1} \sim \chi_{m}^{2}$ and $W_{1}+W_{2} \sim \chi_{m+n}^{2}$, then $W_{2} \sim \chi_{n}^{2}$, one finds that $-2 \log _{e} \lambda \sim \chi_{2 k-2}^{2}$ under $H_{0}$.

The above result should be contrasted to the regular case (when the support of the distribution does not depend on parameters) where under some regularity conditions, $-2 \log _{e} \lambda$ is known to have an asymptotic chisquared distribution. In a similar scenario with $n$ observations and $k$ unknown parameters in general, and 1 under the null, the associated degrees of freedom in the regular case would have been $(n-1)-(n-k)=k-1$ instead of $2(k-1)$.

Empirical Bayes (EB) analysis has, of late, become very popular in statistics, especially when the problem is simultaneous estimation of several parameters. An EB scenario is one in which known relationships among the coordinates of a parameter vector, say, $\theta=\left(\theta_{1}, \cdots, \theta_{k}\right)^{T}$ allow use of the data to estimate some features of the prior distribution. For example, one may have reasons to believe that the $\theta_{i}$ are iid from a prior $\Pi_{0}$, where $\Pi_{0}$ is structurally known except possibly for some unknown parameter (possibly vector-valued) $\lambda$. A parametric EB procedure is one where $\lambda$ is estimated from the marginal distribution of the observations.

Often in an EB analysis, one is interested in finding Bayes risks of the EB estimators. Basu's Theorem helps considerably in many such calculations as we demonstrate below.

Example 7 We consider an EB framework as proposed in Morris (1983a, 1983b). Let $X_{i} \mid \theta_{i}$ be independent $N\left(\theta_{i}, V\right)$, where $V(>0)$ is assumed known. Let $\theta_{i}$ be independent $N\left(z_{i}^{T} \boldsymbol{b}, A\right), i=1, \cdots, k$. The $p$-component $(p<k)$ design vectors $z_{i}$ are assumed to be known, and let $\boldsymbol{Z}^{T}=\left(z_{1}, \cdots, z_{k}\right)$. We assume $\operatorname{rank}(\boldsymbol{Z})=\mathrm{p}$. Based on the above likelihood and the prior, the posteriors of the $\theta_{i}$ are independent $N\left((1-B) X_{i}+B z_{i}^{T} \boldsymbol{b}, V(1-B)\right)$, where $B=V /(V+A)$. Accordingly, the posterior means, the Bayes estimators of the $\theta_{i}$ are given by

$$
\hat{\theta}_{i}^{B A}=(1-B) X_{i}+B z_{i}^{T} \boldsymbol{b}, i=1, \cdots, k .
$$

In an EB set up, $\boldsymbol{b}$ and $A$ are unknown, and need to be estimated from the marginal distributions of the $X_{i}$ 's. Marginally, the $X_{i}$ 's are independent with $X_{i} \sim N\left(z_{i}^{T} \boldsymbol{b}, V+A\right)$. Then, writing $\boldsymbol{X}=$ $\left(X_{1}, \cdots, X_{k}\right)^{T}$, based on the marginal distribution of $\boldsymbol{X}$, the complete sufficient statistic for $(\boldsymbol{b}, A)$ is $\left(\hat{\boldsymbol{b}}, S^{2}\right)$, where $\hat{\boldsymbol{b}}=\left(\boldsymbol{Z}^{T} \boldsymbol{Z}\right)^{-1} \boldsymbol{Z}^{T} \boldsymbol{X}$ is the least squares estimator or the MLE of $\boldsymbol{b}$, and $S^{2}=$ $\sum_{i=1}^{k}\left(X_{i}-z_{i}^{T} \hat{\boldsymbol{b}}\right)^{2}$. Also, based on the marginal of $\boldsymbol{X}, \hat{\boldsymbol{b}}$ and $S^{2}$ are independently distributed with $\hat{\boldsymbol{b}} \sim N\left(\boldsymbol{b},(V+A)\left(\boldsymbol{Z}^{T} \boldsymbol{Z}\right)^{-1}\right)$, and $S^{2} \sim(V+A) \chi_{k-p}^{2}$. Accordingly $\boldsymbol{b}$ is estimated by $\hat{\boldsymbol{b}}$. The MLE 
of $B$ is given by $\min \left(k V / S^{2}, 1\right)$, while its UMVUE is given by $V(k-p-2) / S^{2}$, where we must assume $k>p+2$ for the latter to be meaningful. If instead, one assigns the prior $\Pi(\boldsymbol{b}, A) \propto 1$ as in Morris $(1983 \mathrm{a}, 1983 \mathrm{~b})$, then the HB estimator of $\theta_{i}$ is given by $\hat{\theta}_{i}^{H B}=\left(1-B^{*}\left(S^{2}\right)\right) X_{i}+$ $B^{*}\left(S^{2}\right) z_{i}^{T} \hat{\boldsymbol{b}}$, where $B^{*}\left(S^{2}\right)=\int_{0}^{1} B^{\frac{1}{2}(k-p-2)} \exp \left(-\frac{1}{2 V} B S^{2}\right) d B / \int_{0}^{1} B^{\frac{1}{2}(k-p-4)} \exp \left(-\frac{1}{2 V} B S^{2}\right) d B$. Thus a general EB estimator of $\theta_{i}$ is of the form

$$
\hat{\theta}_{i}=\left[1-\hat{B}\left(S^{2}\right)\right] X_{i}+\hat{B}\left(S^{2}\right) z_{i}^{T} \hat{\boldsymbol{b}}
$$

We will now demonstrate an application of Basu's Theorem in finding the mean squared error (MSE) matrix $E\left[(\hat{\boldsymbol{\theta}}-\boldsymbol{\theta})(\hat{\boldsymbol{\theta}}-\boldsymbol{\theta})^{T}\right]$, where $\hat{\boldsymbol{\theta}}=\left(\hat{\theta}_{1}, \cdots, \hat{\theta}_{k}\right)^{T}$, and expectation is taken over the joint distribution of $\boldsymbol{X}$ and $\boldsymbol{\theta}$. The following theorem provides a general expression for the MSE matrix.

Theorem 5 With the notations of this section,

$$
\begin{aligned}
E\left[(\hat{\boldsymbol{\theta}}-\boldsymbol{\theta})(\hat{\boldsymbol{\theta}}-\boldsymbol{\theta})^{T}\right] & =V(1-B) \boldsymbol{I}_{k}+V B \boldsymbol{Z}\left(\boldsymbol{Z}^{T} \boldsymbol{Z}\right)^{-1} \boldsymbol{Z}^{T} \\
& +E\left[\left(\hat{B}\left(S^{2}\right)-B\right)^{2} S^{2}\right](k-p)^{-1}\left(\boldsymbol{I}_{k}-\boldsymbol{Z}\left(\boldsymbol{Z}^{T} \boldsymbol{Z}\right)^{-1} \boldsymbol{Z}^{T}\right) .
\end{aligned}
$$

Proof Write $\hat{\boldsymbol{\theta}}^{B A}=(1-B) \boldsymbol{X}+B \boldsymbol{Z} \boldsymbol{b}$. Then

$$
\begin{aligned}
E\left[(\hat{\boldsymbol{\theta}}-\boldsymbol{\theta})(\hat{\boldsymbol{\theta}}-\boldsymbol{\theta})^{T}\right] & =E\left[\left(\boldsymbol{\theta}-\hat{\boldsymbol{\theta}}^{B A}+\hat{\boldsymbol{\theta}}^{B A}-\hat{\boldsymbol{\theta}}\right)\left(\boldsymbol{\theta}-\hat{\boldsymbol{\theta}}^{B A}+\hat{\boldsymbol{\theta}}^{B A}-\hat{\boldsymbol{\theta}}\right)^{T}\right] \\
& =E\left[\left(\boldsymbol{\theta}-\hat{\boldsymbol{\theta}}^{B A}\right)\left(\boldsymbol{\theta}-\hat{\boldsymbol{\theta}}^{B A}\right)^{T}\right]+E\left[\left(\hat{\boldsymbol{\theta}}^{B A}-\hat{\boldsymbol{\theta}}\right)\left(\hat{\boldsymbol{\theta}}^{B A}-\hat{\boldsymbol{\theta}}\right)^{T}\right],
\end{aligned}
$$

since

$$
E\left[\left(\boldsymbol{\theta}-\hat{\boldsymbol{\theta}}^{B A}\right)\left(\hat{\boldsymbol{\theta}}^{B A}-\hat{\boldsymbol{\theta}}\right)^{T}\right]=E\left[E\left(\boldsymbol{\theta}-\hat{\boldsymbol{\theta}}^{B A} \mid \boldsymbol{X}\right)\left(\hat{\boldsymbol{\theta}}^{B A}-\hat{\boldsymbol{\theta}}\right)^{T}\right]=0 .
$$

Now

$$
\begin{aligned}
E\left[\left(\boldsymbol{\theta}-\hat{\boldsymbol{\theta}}^{B A}\right)\left(\boldsymbol{\theta}-\hat{\boldsymbol{\theta}}^{B A}\right)^{T}\right] & =E\left[\left(\boldsymbol{\theta}-\hat{\boldsymbol{\theta}}^{B A}\right)\left(\boldsymbol{\theta}-\hat{\boldsymbol{\theta}}^{B A}\right)^{T} \mid \boldsymbol{X}\right] \\
& =E[\operatorname{Var}(\boldsymbol{\theta} \mid \boldsymbol{X})]=E\left[V(1-B) \boldsymbol{I}_{k}\right]=V(1-B) \boldsymbol{I}_{k}
\end{aligned}
$$

Next after a little algebra, we get

$$
\hat{\boldsymbol{\theta}}^{B A}-\hat{\boldsymbol{\theta}}=\left(\hat{B}\left(S^{2}\right)-B\right)(\boldsymbol{X}-\boldsymbol{Z} \hat{\boldsymbol{b}})+B \boldsymbol{Z}(\hat{\boldsymbol{b}}-\boldsymbol{b}) .
$$

Now by the independence of $\hat{\boldsymbol{b}}$ with $\boldsymbol{X}-\boldsymbol{Z} \hat{\boldsymbol{b}}$, noting $S^{2}=\|\boldsymbol{X}-\boldsymbol{Z} \hat{\boldsymbol{b}}\|^{2}$, where $\|\cdot\|$ denotes the Euclidean norm, and $\operatorname{Var}(\hat{\boldsymbol{b}})=V B^{-1}\left(\boldsymbol{Z}^{T} \boldsymbol{Z}\right)^{-1}$, one gets

$$
\begin{aligned}
E\left(\hat{\boldsymbol{\theta}}^{B A}-\hat{\boldsymbol{\theta}}\right)\left(\hat{\boldsymbol{\theta}}^{B A}-\hat{\boldsymbol{\theta}}\right)^{T} & =E\left[\left(\hat{B}\left(S^{2}\right)-B\right)^{2}(\boldsymbol{X}-\boldsymbol{Z} \hat{\boldsymbol{b}})(\boldsymbol{X}-\boldsymbol{Z} \hat{\boldsymbol{b}})^{T}\right] \\
& +V B \boldsymbol{Z}\left(\boldsymbol{Z}^{T} \boldsymbol{Z}\right)^{-1} \boldsymbol{Z}^{T}
\end{aligned}
$$

Next we observe that

$$
(\boldsymbol{X}-\boldsymbol{Z} \hat{\boldsymbol{b}})(\boldsymbol{X}-\boldsymbol{Z} \hat{\boldsymbol{b}})^{T} / S^{2}=\frac{[(\boldsymbol{X}-\boldsymbol{Z} \boldsymbol{b})-\boldsymbol{Z}(\hat{\boldsymbol{b}}-\boldsymbol{b})][(\boldsymbol{X}-\boldsymbol{Z} \boldsymbol{b})-\boldsymbol{Z}(\hat{\boldsymbol{b}}-\boldsymbol{b})]^{T}(V+A)^{-1}}{\|(\boldsymbol{X}-\boldsymbol{Z} \boldsymbol{b})-\boldsymbol{Z}(\hat{\boldsymbol{b}}-\boldsymbol{b})\|^{2}(V+A)^{-1}}
$$


is ancillary, and by Basu's Theorem, is independent of $S^{2}$, which is a function of the complete sufficient statistic $\left(\hat{b}, S^{2}\right)$. Accordingly,

$$
E\left[(\hat{B}(S)-B)^{2}(\boldsymbol{X}-\boldsymbol{Z} \hat{\boldsymbol{b}})(\boldsymbol{X}-\boldsymbol{Z} \hat{\boldsymbol{b}})^{T}\right]=E\left[(\hat{B}(S)-B)^{2} S^{2}\right] E\left[(\boldsymbol{X}-\boldsymbol{Z} \hat{\boldsymbol{b}})(\boldsymbol{X}-\boldsymbol{Z} \hat{\boldsymbol{b}})^{T} / S^{2}\right],
$$

and then by the formula for moments of ratios,

$$
\begin{aligned}
E\left[(\boldsymbol{X}-\boldsymbol{Z} \hat{\boldsymbol{b}})(\boldsymbol{X}-\boldsymbol{Z} \hat{\boldsymbol{b}})^{T} / S^{2}\right] & =E\left[(\boldsymbol{X}-\boldsymbol{Z} \hat{\boldsymbol{b}})(\boldsymbol{X}-\boldsymbol{Z} \hat{\boldsymbol{b}})^{T}\right] / E\left(S^{2}\right) \\
& =(k-p)^{-1}\left[\boldsymbol{I}_{k}-\boldsymbol{Z}\left(\boldsymbol{Z}^{T} \boldsymbol{Z}\right)^{-1} \boldsymbol{Z}^{T}\right] .
\end{aligned}
$$

The theorem follows.

\section{References}

BASU, D. (1955). On statistics independent of a complete sufficient statistic. Sankhya, 15, 377-380.

BASU, D. (1958). On statistics independent of a sufficient statistic. Sankhya, 20, 223-226.

BOOS, D.D. and HUGHES-OLIVER, J.M. (1998). Applications of Basu's Theorem. The American Statistician, 52, 218-221.

HOGG, R.V. and CRAIG, A.T. (1956). Sufficient statistics in elementary distribution theory. Sankhya, 16, $209-216$.

JOHNSTONE, I. M. and VELLEMAN, P.F. (1985). Efficient scores, variance decomposition, and Monte carlo swindles. Journal of the American Statistical Association, 80, 851-862.

KOEHN, U. and THOMAS, D.L. (1975). On statistics independent of a sufficient statistic: Basu's Lemma. American Statistician, 29, 40-42.

LEHMANN, E.L. (1981). An interpretation of completeness and Basu's Theorem. Journal of the American Statistical Association, 76, 335-340.

MORRIS, C. N. (1983a). Parametric empirical Bayes confidence intervals. In Scientific Inference, Data Analysis and Robustness. Eds. G.E.P. Box, T. Leonard and C.F.J. Wu. Academic Press, New York, pp 25-50.

MORRIS, C.N. (1983b) (with discussion). Parametric empirical Bayes inference: theory and applications. Journal of the American Statistical Association, 78, 47-65. 